\title{
Comorbilidade de perturbação de personalidade Borderline e síndroma do cólon irritável: relato de caso a propósito da importância do modelo médico biopsicossocial e da integração de cuidados
}

Cátia Priscila Martins,* Filipa Caetano,* Hugo Almeida, ${ }^{*}$ Serafim Carvalho*

\section{RESUMO}

Introdução: O síndroma do cólon irritável ( $\mathrm{SCl}$ ) é uma perturbação gastrointestinal funcional comum nos países ocidentais, cuja sintomatologia inclui distensão e/ou dor abdominal e alterações do trânsito intestinal, como diarreia e/ou obstipação (critérios de Roma IV). A Perturbação de Personalidade Borderline (PPB) é pautada por instabilidade interpessoal e desregulação afetiva e comportamental. As queixas somáticas são frequentes nos doentes com PPB e a coexistência de sintomas gastrointestinais e PPB parece representar um risco acrescido de suicídio e pior qualidade de vida. A sobreposição destes sintomas e os riscos associados requerem uma abordagem clínica integrada, de modo a garantir um tratamento mais eficaz e menos iatrogénico.

Descrição do caso: Mulher de 31 anos, acompanhada em psicologia desde a infância e com diagnóstico de PPB, sem antecedentes pessoais médico-cirúrgicos de relevo e com antecedentes familiares de $\mathrm{SCl}$. Foi referenciada a consulta de psiquiatria aos 28 anos, por episódio depressivo, sendo medicada com mirtazapina 30mg id (por dia), lamotrigina 100mg 2id e clobazam $20 \mathrm{mg}$ id, com remissão total da sintomatologia depressiva. Cerca de dois anos depois iniciou episódios recorrentes de dor abdominal e dejeções diarreicas, em contexto de assédio moral no local de trabalho. Os exames complementares não apresentavam alterações e os sintomas melhoraram após psicoterapia e mudança de trabalho, apresentando episódios limitados após ingestão de alimentos específicos. Foi feito diagnóstico de $\mathrm{SCl}$.

Comentário: O enquadramento biopsicossocial dos sintomas revelou-se fundamental não só para descobrir uma relação entre o início dos sintomas físicos e os eventos aversivos e iniciar plano de resolução de problemas e de mudança de vida adequado, mas também para estabelecer o diagnóstico de SCl. O diálogo multidisciplinar e uma maior articulação entre a medicina geral e familiar e a psiquiatria são passos fulcrais para a otimização do modelo biopsicossocial e para uma medicina mais humanizada.

Palavras-chave: Perturbação de Personalidade Borderline; Síndroma do cólon irritável; Stress psicológico; Assédio moral.

\section{INTRODUÇÃO}

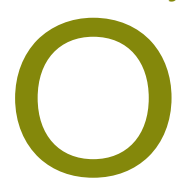

síndroma do cólon irritável (SCI) é uma perturbação gastrointestinal funcional comum nos países ocidentais (10-20\% de prevalência), cuja sintomatologia inclui distensão e/ou dor abdominal e alterações do trânsito intestinal, como diarreia e/ou obstipação. ${ }^{1}$ Estudos sobre a interação entre os sistemas nervoso central e periféricos - autonómico e entérico -, têm contribuído para uma maior compreensão da patogénese do SCI e apontam para uma interação desregulada envolvendo o funcionamento de vias neuronais, endócrinas e imunológicas. Fatores biológicos, psicológicos e ambientais atuam sobre estes sistemas, interagem com fatores de suscetibilidade genética e são tidos como indutores de

*Médicos Internos de Psiquiatria. Serviço B de Internamento de Agudos, Hospital de Magalhães Lemos. 
stress especialmente importantes em fases precoces do desenvolvimento. ${ }^{2}$

A Perturbação de Personalidade Borderline (PPB), apesar da heterogeneidade da sua apresentação clínica, é nuclearmente pautada por instabilidade interpessoal e desregulação afetiva e comportamental, apresentando altas taxas de comorbilidade com outras perturbações mentais. ${ }^{3-4}$ As queixas somáticas são frequentes nos doentes com PPB e a coexistência de sintomas gastrointestinais e PPB parece representar um risco acrescido de suicídio e pior qualidade de vida. ${ }^{5}$ A sobreposição destes sintomas e os riscos associados requerem uma abordagem clínica integrativa, de modo a garantir um tratamento mais eficaz e menos iatrogénico.

Os autores propõem apresentar o relato de um caso de comorbilidade de PPB e SCI, que ilustra os desafios que a complexidade desta associação de diagnósticos coloca à prática clínica.

\section{DESCRIÇÃO DO CASO}

Identificação e história psicossocial

Mulher de 31 anos, raça caucasiana, solteira, natural e residente em Vila Nova de Gaia. Tem o $12^{\circ}$ ano de escolaridade e é funcionária numa lavandaria. Não tem filhos nem irmãos e insere-se numa família monoparental (vive com a mãe, pais divorciados), na fase VI do Ciclo de Vida de Duvall e classe III da Classificação Socioeconómica de Graffar (Figura 1), residindo em habitação com condições higiénico-sanitárias adequadas, sem animais de estimação.

\section{Antecedentes pessoais}

Sem antecedentes médico-cirúrgicos, nem ginecológicos de relevo (nulípara). Plano nacional de vacinação atualizado. Pratica regularmente aulas de dança. Dieta alimentar padrão, sem restrições. Consumo esporádico de álcool, de tabaco, de canabinoides e de bebidas que contêm xantinas.

\section{Antecedentes familiares}

A mãe da utente tem 56 anos e diagnóstico de SCI, com episódios de diarreia e dor abdominal, sobretudo em momentos de maior ansiedade. O pai tem 59 anos e diagnóstico de epilepsia, tendo iniciado sintomas aos 14 anos e permanecido medicado até aos 44 , estando sem crises desde os 39 anos; segundo a utente "sempre teve tendência para episódios de diarreia" (sic), mas esses episódios nunca foram explorados do ponto de vista médico, nem causaram impacto funcional significativo (Figura 1).

O tio paterno, com 56 anos, tem patologia cardiovascular, que a utente não sabe precisar (Figura 1).

O avô paterno faleceu aos 86 anos por complicações de doença de Alzheimer e a avó paterna aos 84 anos por enfarte agudo do miocárdio. $\mathrm{O}$ avô materno faleceu antes do nascimento da utente, aos 49 anos, por cancro do pulmão, e a avó materna aos 84 anos devido a complicações da doença de Alzheimer (Figura 1).

Um primo do lado materno com antecedentes de toxicodependência, abstinente nos últimos 10 anos de vida, faleceu num acidente de trabalho aos 43 anos (Figura 1).

\section{Antecedentes de saúde mental}

Acompanhamento em consulta de psicologia, em regime privado, desde os sete anos de idade. A utente desconhece o motivo de início deste acompanhamento, mas descreve ter sofrido bullying na escola e iniciado, na adolescência, comportamentos autolesivos e consumo abusivo de álcool e canabinoides, apresentando, desde essa altura, sentimentos de vazio e instabilidade relacional. Os comportamentos autolesivos ocorreram dos 12 aos 19 anos e depois novamente aos 21 anos (após rutura amorosa). Diagnóstico não formal sugestivo de PPB (avaliação de psicologia).

Passou a ser acompanhada por psiquiatria aos 27 anos, referenciada pelo médico de família, por humor depressivo. Referiu falecimento recente de avó paterna e primo materno (relações muito próximas). Foi despedida do restaurante onde trabalhava, o que atribuiu a "não andar bem emocionalmente" (sic). Nos sete meses que se seguiram ao seu despedimento descreveu isolamento social e desenvolvimento progressivo de sintomatologia depressiva, com humor depressivo, anorexia com perda ponderal de $2 \mathrm{~kg}$ (de $48 \mathrm{~kg}$ para $46 \mathrm{~kg}$ ), anedonia, ideias de morte recorrentes, insónia inicial e noção de sono pouco reparador, com pesadelos frequentes (deixou de dormir no quarto, para evitar memórias dos entes perdidos). Na primeira consulta de psiquiatria concluiu-se que a sintomatologia era compatível com episódio depressivo, tendo-se medicado com mirtazapina 30mg e clobazam 20mg por dia. Após 


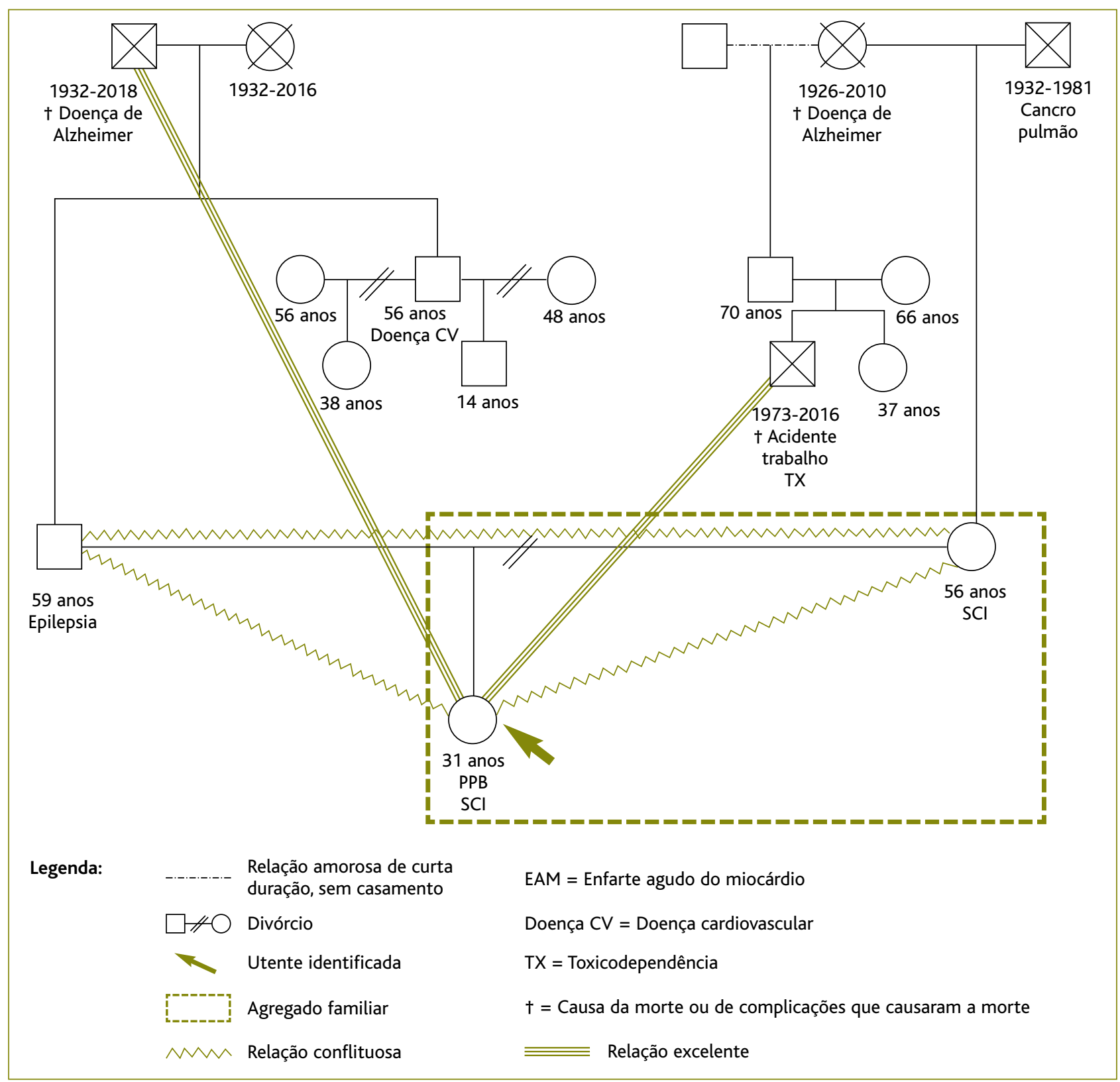

Figura 1. Genograma.

seis semanas apresentava-se na consulta eutímica, com ganho ponderal de $3 \mathrm{~kg}$ e sono regularizado (capaz de dormir novamente no seu quarto). Pela sonolência diurna, o clobazam foi gradualmente titulado até à total suspensão.

As consultas seguintes confirmaram a hipótese diagnóstica de $\mathrm{PPB}$, não só pelos antecedentes pessoais, mas também pela marcada instabilidade relacional e pelas dificuldades de regulação emocional que se mantiveram mesmo após resolução do episódio depressivo (Modelo de Mentalização de Bateman e Fonagy). ${ }^{6}$ Foi proposta introdução de lamotrigina como adjuvante terapêutico (100mg 2id). Retomou a atividade laboral cerca de 10 meses após a primeira consulta (estágio 
remunerado em loja) e ampliou gradualmente o seu círculo social. A instabilidade relacional mais significativa verificou-se sobretudo na sua relação com os pais e no domínio das relações amorosas, com tendência à dicotomia idealização/desidealização e ao envolvimento em relações abusivas ou com indivíduos rejeitantes, reforçadores das suas cognições de abandono. O autocriticismo e elevados níveis de vergonha destacaram-se sempre como importantes fatores de agravamento do seu sofrimento, mais intolerável após episódios de abandono/rejeição, tendo inclusive uma das ruturas amorosas culminado na ingestão medicamentosa voluntária de nove comprimidos de cloxazolam $2 \mathrm{mg}$ (da mãe), de modo impulsivo e com intuito de frenar a dor da vivência de abandono.

Da sua história de desenvolvimento pessoal destacam-se uma família nuclear disfuncional, com conflitos frequentes entre os pais e agressividade verbal e física do pai sobre a mãe, presenciada pela utente durante a infância e a adolescência. Os pais separam-se quando tinha 14 anos, com várias tentativas de reconciliação posteriormente, de curta duração e com ruturas conflituosas, culminando em divórcio quando tinha 27 anos. A relação com os pais foi sempre muito instável, denotando a sua vivência caracteristicamente polarizada do mundo e das relações interpessoais, com tendência a oscilar entre a idealização e a desidealização, o que se refletiu em períodos de corte total de contacto com um deles, geralmente com idealização do outro, seguidos do processo inverso, sendo esta alternância geralmente despoletada por uma vivência de abandono do progenitor idealizado, real ou experienciada como tal. A descrição dos pais, segundo os relatos da utente, faz supor tratar-se de um casal com uma relação de dominância/subjugação, sendo o pai autoritário, dominante e controlador, a mãe submissa e com traços dependentes de personalidade e sendo ambos manipuladores, muitas vezes culpabilizando a utente pelas suas dificuldades conjugais. Esta dinâmica familiar e estilos parentais sugerem experiências continuadas de privação emocional e vergonha, que a utente terá vivenciado desde idades precoces e que se mantêm até ao presente, não só na relação com os pais, mas na sua experiência interna de si, do mundo e dos outros, traduzindo-se numa visão altamente autocrítica (com comportamentos e diálogo interno autopunitivos) e numa tendência a relações interpessoais que perpetuam estes padrões de abuso, rejeição, subjugação e privação emocional iniciados em idades precoces.

\section{História da doença atual e evolução clínica}

Em contexto de consulta programada de acompanhamento de psiquiatria (agosto 2018), a utente referiu como queixa principal dor abdominal, tendo facultado informação clínica remetida pelo médico de família, descrevendo o quadro clínico de queixas gastrointestinais de difícil controlo, em utente com possível diagnóstico SCI, para eventual integração dos sintomas na psicopatologia de base.

A dor iniciara nos últimos quatro meses e localizava-se nos quadrantes inferiores do abdómen, por vezes com irradiação epigástrica, com características de cólica e sem fatores de alívio ou agravamento identificáveis. Concomitantemente apresentava dejeções diarreicas, que ocorriam em média quatro a cinco vezes por dia, por vezes aquosas, com alguns episódios de oito a dez dejeções por dia (em que recorreu ao serviço de urgência). Negava febre, vómitos e dejeções com sangue ou noturnas ao longo de toda a evolução.

Nesse período, nos últimos quatro meses, recorreu oito vezes a consulta médica não programada (cuidados de saúde primários e hospitalares), tendo realizado exames complementares de diagnóstico. Em dois desses episódios foi administrada medicação para alívio sintomático (paracetamol 1000mg, metoclopramida $10 \mathrm{mg}$, ranitidina $150 \mathrm{mg}$ ), tendo tido alta com melhoria e/ou remissão total das queixas.

O estudo diagnóstico realizado, nomeadamente pelo médico de família, incluiu: estudo analítico (hemograma, TSH, ionograma, proteína $\mathrm{C}$ reativa, enzimas hepáticas, bilirrubina, creatinina, ureia, albumina, lípase, sumária de urina tipo II), radiografia abdominal, ecografia abdominal e colonoscopia. Os exames não apresentaram alterações significativas. Não foram constatadas alterações no exame físico, nem variações no IMC da utente $\left(17,6 \mathrm{~kg} / \mathrm{m}^{2}\right)$.

Na avaliação clínica (psiquiatria, agosto 2018), a utente não apresentava alterações das funções cognitivas nem alterações significativas do exame físico. Revelava sentir-se muito ansiosa nos últimos meses, coincidente com problemática laboral e relacional. Referia sofrer de assédio moral por parte da entidade patronal, 
progressivamente mais frequente, com atribuição de funções de difícil execução sem apoio de outros funcionários, ameaças de despedimento e comentários de desvalorização do seu trabalho e pessoa. Tinha terminado recentemente uma relação amorosa, de cerca de um mês, abusiva e com episódios recorrentes de agressividade verbal, culminando em episódio de agressão física, que levou a utente a terminar a relação.

Nas três consultas sucessivas estabeleceu-se a estratégia de escuta ativa, validação e diálogo socrático. A utente identificou emoções de medo e de raiva relativamente à sua chefia e ao seu namorado, associadas a autocriticismo e raiva autodirigida. Identificou a possibilidade de associação dos sintomas intestinais com o seu sofrimento emocional. Não equacionou deixar imediatamente o trabalho por razões financeiras, considerando, no entanto, procurar outro emprego.

Duas semanas após estas três consultas (outubro 2018), a utente compareceu no serviço de psiquiatria em que é acompanhada, sem marcação, apelativa, referindo que veio diretamente do trabalho "por não aguentar mais e que se permanecesse lá não sabia o que teria feito" (sic). Relatava agravamento da tensão a nível laboral, sentindo-se constantemente ansiosa, com insónia, anorexia, choro fácil, dificuldades de concentração, tendência ao isolamento e pensamentos constantes sobre o trabalho, associados a sentimentos de desesperança. Os episódios de diarreia e dor abdominal tinham-se intensificado e referia marcada ansiedade antecipatória relacionada com o trabalho, sobretudo ao final do dia e pela manhã, a caminho do local. Nesse dia, após novo episódio de tensão com a chefia, sentiu raiva intensa e "medo de a agredir" (sic), tendo optado por recorrer ao serviço de psiquiatria. Após validação do sofrimento e reflexão sobre os últimos eventos sucedidos na sua vida foi recomendado retomar clobazam (20mg ao deitar) e manter-se temporariamente afastada do trabalho, por estar incapaz de manter funcionamento laboral adequado e por se entender que este constituía condicionante importante para os sintomas apresentados.

Uma semana após a última ocorrência, a utente apresentava sono e apetite regularizados, diminuição da ansiedade, melhoria da abdominalgia e diminuição significativa da frequência de dejeções diarreicas, estando determinada a abandonar o emprego em que se encontrava. Manteve-se um mês com acompanhamento semanal em consulta de psiquiatria, estabelecendo-se plano de ativação comportamental, incluindo procura de novo emprego. Em novembro de 2018 retomou as funções laborais por três dias, ao fim dos quais se despediu, por ter recebido proposta de emprego como assistente operacional num hospital. Por estabilização do quadro, estabeleceu-se a redução da periodicidade das avaliações clínicas e redigiu-se informação clínica destinada ao médico de família, com a avaliação e a estratégia clínica consideradas, com indicação de gestão sintomática e de promoção de estilos de vida que favoreçam o regular funcionamento gastrointestinal e com recomendação de pedido de reavaliação antecipada por psiquiatria em caso de recidiva de sintomas com impacto significativo na qualidade de vida da utente.

Pela sintomatologia, exclusão de outras patologias e tempo de evolução considerou-se o diagnóstico de SCI em utente com PPB. Até à última avaliação por psiquiatria (março de 2019), a utente manteve-se estável (sob mirtazapina 30mg, lamotrigina 100mg 2id), satisfeita com o novo emprego, referindo marcada diminuição da frequência das queixas de dor abdominal e de diarreia (sob butilescopolamina 10mg e paracetamol $100 \mathrm{mg}$ em SOS, com cuidados alimentares adequados).

\section{COMENTÁRIO}

Apesar dos estudos que relacionam características de personalidade e SCI não permitirem desenhar conclusões firmes, parece haver alguma relação entre SCI e características como a alexitimia, elevada emocionalidade negativa e hipervigilância das sensações corporais e/ou interpretação distorcida da sua relação com os estados emocionais. ${ }^{1-2}$ As dificuldades de mentalização e de regulação emocional encontradas em doentes com PPB são compatíveis com algumas destas características. $^{3-6}$

A escassez de estudos sobre a comorbilidade de PPB e SCI contrasta com a convergência de ambos os diagnósticos para processos de regulação emocional que poderão ter aspetos interligados ou partilhados: sendo o stress um importante fator de exacerbação no SCI, a instabilidade emocional característica da $\mathrm{PPB}$ pode tornar estes doentes mais vulneráveis a exacerbações, podendo mesmo as queixas físicas ser encaradas como 
manifestação das dificuldades de autorregulação características da PPB. Por outro lado, esta co-ocorrência pode dever-se a fatores de risco comuns a ambas (como experiências precoces adversas de negligência e abuso), sendo frequente encontrar crenças sobre a inaceitabilidade de emoções negativas quer em doentes com SCI, quer em doentes com PPB, possivelmente como consequência de terem crescido em ambientes em que a expressão dessas emoções foi punida ou ignorada. Por último, pode haver uma base biológica comum, visto que em ambos parece haver envolvimento da serotonina e de alterações do eixo-hipotálamo-hipofisáriosuprarrenal. ${ }^{5,7}$

O diagnóstico de SCI é essencialmente clínico. No caso relatado, as características clínicas apresentadasdor abdominal e dejeções diarreicas recorrentes, sem sangue, vómitos ou febre--, a par da história familiar de SCI (mãe com diagnóstico de SCI e pai com episódios frequentes de diarreia que, apesar de não ter diagnóstico de SCI, leva a ponderar a possibilidade de estar subdiagnosticado ou de ter alguma vulnerabilidade passível de transmissão genética), apontavam para esta hipótese diagnóstica.

O desafio clínico colocava-se na gravidade dos sintomas (que interferiam significativamente com o funcionamento global da utente), no seu aspeto emagrecido e nos seus períodos de anorexia. O IMC, apesar de baixo, apresentou-se sempre dentro dos valores habituais para a doente. É, contudo, compreensível que numa abordagem em contexto de urgência, o aspeto emagrecido possa ser encarado como sinal de alarme, juntamente com a anorexia, sintoma de ansiedade habitual na utente em alturas de maior stress psicológico, mas que sem uma abordagem detalhada dos aspetos emocionais, é facilmente considerado sugestivo de patologia intestinal orgânica. Não existiam outros sinais de alarme (sinais cuja presença deve motivar a realização de exames complementares para despite de doença intestinal orgânica); porém, também não existiam muitas das manifestações típicas da doença (Tabelas 1 e 2) ${ }^{8-9}$ Por outro lado, segundo os critérios de Roma IV, o diagnóstico só poderia estabelecer-se após pelo menos seis meses do início dos sintomas (Tabela 3)..$^{8-9}$

A abordagem de aspetos emocionais e o enquadramento biopsicossocial dos sintomas revelou-se fundamental para descobrir uma relação temporal entre o

\section{TABELA 1. Características típicas do $\mathrm{SCl}^{8}$}

Dejeções moles/frequentes

Obstipação

Distensão abdominal

Dor abdominal

Sintomas associados à ingestão alimentar e/ou a sensibilidades alimentares específicas

Sintomas variáveis ao longo do tempo (e.g., padrão de dejeções, localização da dor)

TABELA 2. Sinais de alarme para patologia intestinal orgânica $^{8}$

Início de sintomas após os 50 anos

Sintomas graves ou agravamento progressivo dos sintomas

Perda de peso inexplicada

Diarreia noturna

História familiar de doença intestinal orgânica (doença celíaca, doença inflamatória intestinal, cancro do cólon)

Melenas ou perda hemática retal

Anemia por défice de ferro sem causa identificada

TABELA 3. Critérios de Roma IV para diagnóstico $\mathrm{SC}^{8 *}$

Dor abdominal recorrente, em média 1 vez/semana nos últimos 3 meses, associada a dois ou mais dos seguintes critérios:

1 - Relação com defecação

2 - Associação a mudança de frequência das dejeções

3 - Associação a mudança de consistência das dejeções

Legenda: ${ }^{*}$ Os critérios devem estar presentes nos últimos três meses, com início dos sintomas pelo menos seis meses antes de se estabelecer o diagnóstico.

início dos sintomas físicos e os eventos indutores de stress, algo que não era, até então, claro para a utente e que é compreensível, considerando as dificuldades características dos doentes com PPB em identificar e contextualizar emoções. Depois de estabelecida esta relação, conseguiu-se iniciar um plano de resolução de problemas adequado e o devido suporte psicológico. $\mathrm{O}$ 
afastamento temporário do local de trabalho, onde a utente sofria diariamente abuso emocional, foi não só importante para diminuir a ansiedade para níveis em que fosse possível a doente perspetivar diferentes alternativas de resolução da sua situação laboral e trabalhar possíveis obstáculos externos e internos (psicológicos), mas também para atestar a relação entre o stress laboral e os sintomas físicos. O desaparecimento dos sintomas quando a utente se afastou do trabalho, local de tensão, abuso e desvalorização repetidos, corrobora o papel da sua vivência emocional aversiva no aparecimento dos sintomas de foro gastrointestinal, seja como precipitante, seja como agravante, sendo reconhecida esta relação nas crises de SCI. ${ }^{1,8}$ Após a demissão e o início de novo trabalho, as crises confinaram-se a episódios muito esporádicos, que a utente associa a consumos de laticínios e carnes vermelhas. Apesar de ainda não haver consenso quanto ao papel da dieta na evolução do SCI, parece ser frequente os doentes reportarem sensibilidade ou intolerância a certos alimentos, com cerca de $90 \%$ a fazerem restrições alimentares, embora as verdadeiras alergias alimentares sejam raramente encontradas. ${ }^{8}$

O elevado recurso aos cuidados de saúde primários e serviço de urgência e a realização de inúmeros exames complementares de diagnóstico, incluindo exames invasivos, tornaram o processo mais ansiogénico para a utente e atrasaram a realização do diagnóstico definitivo e uma intervenção terapêutica adequada.

Uma abordagem holística, integrando as dimensões biológica, psicológica e social do utente, garante uma avaliação mais acurada e menos invasiva e uma intervenção mais eficaz e menos iatrogénica. Neste sentido, os cuidados de saúde primários têm um lugar de destaque pela proximidade ao utente, família e comunidade, tendo o médico de família um papel central na integração de conhecimentos clínicos de diversas áreas da medicina e na comunicação com as várias especialidades.

A implementação de medidas institucionais e organizativas que facilitem o diálogo multidisciplinar e uma maior articulação dos cuidados de saúde, particularmente entre a medicina geral e familiar e a psiquiatria e saúde mental, são passos fulcrais para a otimização do modelo biopsicossocial e para uma medicina mais humanizada.

\section{REFERÊNCIAS BIBLIOGRÁFICAS}

1. Fournier A, Mondilon L, Dantzer C, Gauchez AS, Ducros V, Mathieu N, et al. Emotional overactivity in patients with irritable bowel syndrome. Neurogastroenterol Motil. 2018;30(10):e13387.

2. Muscatello MR, Bruno A, Mento C, Pandolfo G, Zoccali RA. Personality traits and emotional patterns in irritable bowel syndrome. World J Gastroenterol. 2016;22(28):6402-15.

3. Leichsenring F, Leibing E, Kruse J, New AS, Leweke F. Borderline personality disorder. Lancet. 2011;377(9759):74-84.

4. Foxhall M, Hamilton-Giachritsis C, Button K. The link between rejection sensitivity and borderline personality disorder: a systematic review and meta-analysis. Br J Clin Psychol. 2019;58(3):289-326.

5. Niesten IJ, Karan E, Frankenburg FR, Fitzmaurice GM, Zanarini MC. Prevalence and risk factors for irritable bowel syndrome in recovered and non-recovered borderline patients over 10 years of prospective followup. Personal Ment Health. 2014;8(1):14-23.

6. Bateman A, Fonagy P. Psychotherapy for Borderline personality disorder:mentalization-based treatment. New York: Oxford University Press; 2004. ISBN 9780198527664

7. Rimes KA, Chalder T. The Beliefs about Emotions Scale: validity, reliability and sensitivity to change. J Psychosom Res. 2010;68(3):285-92.

8. Chey WD, Kurlander J, Eswaran S. Irritable bowel syndrome: a clinical review. JAMA. 2015;313(9):949-58.

9. Schmulson MJ, Drossman DA. What is new in Rome IV. J Neurogastroenterol Motil. 2017;23(2):151-63.

\section{CONFLITO DE INTERESSES}

Os autores declaram não ter quaisquer conflitos de interesse.

\section{FINANCIAMENTO}

O trabalho apresentado neste manuscrito não foi objeto de qualquer tipo de financiamento externo (incluindo bolsas e investigação).

\section{ENDEREÇO PARA CORRESPONDÊNCIA}

Cátia Priscila Martins

E-mail: catiapriscilamartins@gmail.com

https://orcid.org/0000-0003-4974-1881

Recebido em 05-08-2019

Aceite para publicação em 03-10-2019 


\section{ABSTRACT}

\section{COMORBIDITY OF BORDERLINE PERSONALITY DISORDER AND IRRITABLE BOWEL SYNDROME: CASE REPORT ON THE IMPORTANCE OF THE MEDICAL BIOPSYCHOSOCIAL MODEL AND THE INTEGRATION OF CARE}

Introduction: Irritable bowel syndrome (IBS) is a common functional gastrointestinal disorder in occidental countries, with symptoms including abdominal pain and/or bloating and alteration of bowel transit, such as diarrhea and/or constipation (Rome IV Criteria). Borderline Personality Disorder (BPD) is driven by interpersonal instability and affective and behavioral dysregulation. Somatic complaints are frequent in patients with BPD and the coexistence of gastrointestinal symptoms and BPD seems to represent an additional risk in terms of suicide and poorer quality of life. The overlap of these symptoms and the associated risks require an integrative clinical approach, in order to grant a more effective and less iatrogenic treatment.

Case description: Woman, 31 years old, followed-up in psychology since childhood, without personal relevant medical history and with a family history of IBS. She started psychiatry appointments for a depressive episode at the age of 28 and was then medicated with mirtazapine $30 \mathrm{mg}$ id, lamotrigine $100 \mathrm{mg} 2 \mathrm{id}$, and clobazam $20 \mathrm{mg}$ id, with total remission of depressive symptoms. Around two years later, she started having recurrent abdominal pain and diarrhea, at a time when she was experiencing bullying in the workplace. The diagnostic tests showed no alterations and her symptoms improved after psychotherapy and after she changed her workplace, with episodes then limited to times after she ingested specific food. IBS was diagnosed.

Comment: The biopsychosocial framing of the symptoms is fundamental not only to find a relationship between the beginning of the physical symptoms and the aversive events and to the starting of a problem-solving plan and adequate life changes but also to establish the diagnosis of IBS. The multidisciplinary dialogue and a better care discussion between the general physician and psychiatrists are central steps to the optimization of the biopsychosocial model and a more humanized medicine.

Keywords: Borderline Personality Disorder; Irritable bowel syndrome; Psychological stress; Workplace bullying. 